\title{
Who is likely to develop persistent low back pain? A longitudinal analysis of prognostic occupational factors
}

\author{
Markus Melloh ${ }^{\mathrm{a}, *}$, Achim Elfering ${ }^{\mathrm{b}}$, Tasha R. Stanton ${ }^{\mathrm{c}, \mathrm{d}}$, Anja Käser ${ }^{\mathrm{b}}$, Cornelia Rolli Salathéb \\ Thomas Barz ${ }^{\mathrm{e}}$, Christoph Röder ${ }^{\mathrm{f}}$ and Jean-Claude Theis ${ }^{\mathrm{g}}$ \\ ${ }^{a}$ QEII Medical Centre, Hospital Avenue, Western Australian Institute for Medical Research (WAIMR), University \\ of Western Australia, Nedlands, WA, Australia \\ ${ }^{\mathrm{b}}$ Department of Work and Organizational Psychology, Institute of Psychology, University of Berne, Berne, \\ Switzerland \\ ${ }^{\mathrm{c}}$ Sansom Institute for Health Research, University of South Australia, Adelaide, Australia \\ ${ }^{\mathrm{d}}$ Neuroscience Research Australia, Sydney, Australia \\ e Department of Orthopaedic Surgery, Asklepios Klinikum Uckermark, Schwedt/Oder, Germany \\ ${ }^{\mathrm{f}}$ Institute for Evaluative Research in Medicine, MEM Research Center, University of Berne, Berne, Switzerland \\ ${ }^{\mathrm{g}}$ Centre for Musculoskeletal Outcomes Research (CMOR), Dunedin School of Medicine, University of Otago, \\ Dunedin, New Zealand
}

\section{Received 20 October 2011}

Accepted 31 August 2012

\begin{abstract}
.
OBJECTIVE: Occupational low back pain (LBP) is considered to be the most expensive form of work disability, with the socioeconomic costs of persistent LBP exceeding the costs of acute and subacute LBP by far. This makes the early identification of patients at risk of developing persistent LBP essential, especially in working populations. The aim of the study was to evaluate both risk factors (for the development of persistent LBP) and protective factors (preventing the development of persistent LBP) in the same cohort.

PARTICIPANTS: An inception cohort of 315 patients with acute to subacute or with recurrent LBP was recruited from 14 health practitioners (twelve general practitioners and two physiotherapists) across New Zealand.

METHODS: Patients with persistent LBP at six-month follow-up were compared to patients with non-persistent LBP looking at occupational, psychological, biomedical and demographic/ lifestyle predictors at baseline using multiple logistic regression analyses. All significant variables from the different domains were combined into a one predictor model.

RESULTS: A final two-predictor model with an overall predictive value of $78 \%$ included social support at work (OR 0.67 ; 95\% CI 0.45 to 0.99 ) and somatization (OR 1.08 ; 95\% CI 1.01 to 1.15 ).

CONCLUSIONS: Social support at work should be considered as a resource preventing the development of persistent LBP whereas somatization should be considered as a risk factor for the development of persistent LBP. Further studies are needed to determine if addressing these factors in workplace interventions for patients suffering from acute, subacute or recurrent LBP prevents subsequent development of persistent LBP.
\end{abstract}

Keywords: Inception cohort study, work-related factors, prognosis, resources, risk factors

\footnotetext{
${ }^{*}$ Corresponding author: Dr. Markus Melloh, Research Associate Professor, Western Australian Institute for Medical Research (WAIMR), University of Western Australia, B Block, QEII Medi-
}

cal Centre, Hospital Avenue, Nedlands, WA 6009, Australia. Tel.: +61 89346 3464; Fax: +61 89346 1818; E-mail: markus.melloh@ uwa.edu.au. 


\section{Introduction}

Occupational musculoskeletal disorders are the most expensive form of work disability [1]. Among these musculoskeletal disorders, low back pain (LBP) is the most common type of musculoskeletal disorder. Socioeconomic costs of persistent LBP exceed the costs of acute $(<$ six weeks) to subacute LBP (six to twelve weeks) and of recurrent LBP by far [2]. Recurrent LBP was defined according to Stanton et al. as at least two episodes of LBP over the past year, with each episode of LBP lasting at least 24 hours, with at least 30 LBPfree days between two episodes, and reported LBP exceeding $20 \mathrm{~mm}$ out of $100 \mathrm{~mm}$ on the Visual Analogue Scale (VAS) [22]. Patients whose VAS scores (of pain intensity during the past week) were greater than $20 \mathrm{~mm}$ at both baseline and six months and whose VAS change scores (between baseline and six-month follow-up) were less than $20 \mathrm{~mm}$ were considered to have persistent LBP. According to a review on pain assessment instruments, a VAS score of less than ten $\mathrm{mm}$ can be interpreted as no pain [26,27]. Therefore, patients with a VAS score $\leqslant 20 \mathrm{~mm}$ at six-month followup were considered to have only minimal pain and thus were not considered to have persistent pain. Hence, identifying patients at an early stage who are at risk of developing persistent LBP is imperative, particularly in working populations [3]. Perhaps of most importance is identifying modifiable risk and protective factors that, if addressed prior to the development of persistent LBP, could limit the associated socioeconomic burden $[4,5]$.

The relevance of occupational factors as risk factors of persistent LBP is controversial. In a systematic review on occupational psychological factors, Linton reported a positive correlation with future LBP episodes and associated functional limitation at work, such as prolonged work absenteeism [6]. From the eleven occupational psychological factors investigated for their association with future LBP, Linton found strong evidence for the following six factors: job satisfaction, monotonous tasks, work relations, work demands, stress and perceived ability to work. The review revealed moderate evidence for work pace, control, emotional effort at work and the belief that work is dangerous. Work content showed inconclusive evidence as factor associated with future LBP.

Two reviews identified the following occupational factors as risk factors for a recovery from an acute LBP episode [7,8]: high task demands (time pressure, job insecurity, interruptions), low control (time and work method), low social support at work, low recognition, unfavourable working environment, work dissatisfaction, belief, that work causes LBP and negative expectations regarding return to work.

In contrast to these reviews, Hartvigsen et al., in their review on prospective cohort studies did not find any evidence for a correlation between occupational psychological factors and LBP or functional limitation due to LBP [9]. However, studies in this field of research are subject to a great variation in methods, factors analysed and assessment instruments [9], which makes it difficult to compare findings from different reviews without clear definitions and guidelines.

To enhance the comparability of cohort studies the Multinational Musculoskeletal Inception Cohort Study (MMICS) Statement recommends the use of internationally accepted core outcome measures [10]. MMICS is a consensus statement with the aim to enhance the quality of prospective cohort studies investigating the transition of early stages of LBP to persistent LBP. The MMICS steering group comprised research experts in occupational, psychosocial and general practice aspects of LBP. The MMICS Statement focused on a core set of predictor factors and included recommendations for appropriate measurement instruments and recommendations about follow-up measurement and timing. Further, occupational factors have recently been demonstrated to be reliable prognostic factors for persistent LBP and thus should be included as part of a minimum set of prognostic measures [11]. Therefore, the current study focused on occupational factors as well as possible psychological, biomedical and demographic/lifestyle influences.

Systematic reviews have investigated the evidence on occupational [9], psychological [12], biomedical [5] and lifestyle risk factors of persistent LBP [13]. However, studies investigated by these reviews often did not evaluate both risk factors (for the development of persistent LBP) and protective factors (preventing the development of persistent LBP) in the same cohort. Another limitation of many studies is that they do not monitor pain across multiple follow-ups. The aim of the current study, is in hypothesizing that development of persistent LBP would relate to both risk and protective factors in the occupational, psychological, biomedical and demographic/lifestyle domains. Factors were considered as risk or protective factors according to these systematic reviews. Including four follow-ups and following the conclusions from the systematic reviews mentioned above, we specifically hypothesized that: 
1) A resigned attitude towards the job, uncertainty, high concentration requirements, work organisational problems and interruptions, time pressure, emotion suppression, work absenteeism [14] as well as single-sided physical stress would be occupational risk factors [6-8,15] for developing persistent LBP, while social support at work, job control and job satisfaction would be protective factors $[6,15]$;

2) Depression and somatisation [16], fear-avoidance and catastrophizing [17] would be psychological risk factors for developing persistent LBP;

3) Pain intensity and duration of LBP [17], radiating pain below the knee [5] and functional limitation would be biomedical risk factors for developing persistent LBP, whereas a good physical health and mental well-being would be protective factors;

4) Higher age, body mass index (BMI), and smoking [18] would be demographic/lifestyle risk factors, whereas being married and physically active [19] would be protective factors.

\section{Material and methods}

This is a prospective cohort study that consists of baseline measurement and follow-up measurements at three, six, and twelve weeks and at six months. The study adhered to the recommendations of the Declaration of Helsinki (2008) and was approved by the local Lower South Regional Ethics Committee (LRS/08/03/008). The protocol for our study has been published previously [20]. The methods are described in brief below.

An inception cohort of 315 patients was consecutively recruited from 14 health practitioners (twelve general practitioners and two physiotherapists) across New Zealand. Participants were asked to take part in the study when attending a health practitioner for their first episode of acute to subacute LBP or for recurrent LBP.

To be included in the present study, patients had to provide written consent, be able to read and write in English, and be between 18 and 65 years of age. Patients were excluded if they had chronic LBP (LBP $>$ twelve weeks at the time of the first visit to a health practitioner) [21,22], specific LBP (infection, tumour, osteoporosis, ankylosing spondylitis, fracture, deformity, inflammatory process, cauda equina syn- drome) [23], a severe comorbidity influencing overall well-being (e.g. painful disabling arthritic hip joints), or if they no longer had LBP at the time of the screening interview. Additionally, patients were excluded if they were currently pregnant or were unwilling to complete questionnaires.

A standardised, structured telephone interview was used to screen potential participants. Eligible patients were then sent a baseline questionnaire by mail and asked to return it within one week. Follow-up questionnaires were sent after three, six, and twelve weeks and at six months. If any questionnaires were not returned, a reminder was sent out after one and two weeks. All questionnaires were based on the recommendations of the MMICS Statement [10] addressing the four domains of occupational, psychological, biomedical and demographic/lifestyle risk factors for the development of persistent LBP and resources preventing persistent LBP. All areas (clinical; financial; lifestyle; psychological; social, demographic, and work) and all measurements recommended by the MMICS Statement were assessed except for the duration on current benefits at baseline and blood tests and functional magnetic resonance imaging at follow-up (for specific factors see Tables 1, 2 and 3). The follow-up guidelines were adhered to except for health care utilization which was only measured once (at six-month follow-up) instead of every two weeks. Participants received \$NZ10 grocery, fuel or book vouchers for each returned questionnaire as compensation for their time.

\subsection{Statistical analysis}

Patients with persistent LBP at six-month follow-up were compared to patients with non-persistent LBP. To determine whether change in VAS score over time was linear, a trend analysis including all time points was completed for persistent and non-persistent LBP, and the difference between groups tested using an independent sample t-test. We conducted an analysis of variance including the repeated measurement factor (baseline and all follow-ups) and a between factor (persistent vs. non-persistent). We tested the interaction of fixed effect polynomial linear trend factor and between factor to be significant indicating that linear decrease of VAS across time was restricted to the non-persistent group while there was no change in VAS in the persistent group.

Having established the distinctness of the two groups, the next step in the approach to statistical analysis was to calculate, the correlation between the variables of 
the four domains and the presence of persistent LBP at six-month follow-up (defined by the VAS score) using Pearson's correlation coefficient. Following this, multiple logistic regression analysis was performed separately for each of the four domains measured at baseline using LBP status at six-month follow-up as the dependent variable (persistent/non-persistent) to identify those domain predictive factors that showed unique prognostic value within their domain. The four domains were: occupational, psychological, biomedical and demographic/lifestyle, and were controlled in the multiple logistic regression analysis for age, gender and body mass index. The overall predictive value was calculated for the full regression models of the different domains. Finally, all significant variables from the different domains were combined into a final predictor model. Data was analyzed using IBM SPSS Statistics 19 (IBM Corp., Armonk, NY, USA). Statistical significance was set at the $P<0.05$ level, two-tailed.

\section{Results}

From April 2008 to October 2010, 562 consecutive patients with acute, subacute or recurrent LBP were screened. One-hundred-and-twenty-four patients were excluded for the following reasons: they were LBPfree at the time of the screening interview (ten); had chronic LBP for more than twelve weeks (93); had specific LBP (eight); had osteoarthritis of the hip or knee joint (two); were pregnant (three); were not available for follow-ups (two) or were above 65 years of age (six). Twenty-six patients declined to participate and 97 patients did not return the baseline questionnaire despite two reminders. Three-hundred-and-fifteen patients were enrolled with 147 patients lost to followup. This resulted in 168 patients participating over the six-month period. Table 1 depicts baseline characteristics of participants who completed six-month followup versus participants lost to follow-up for areas recommended by the MMICS Statement. Baseline characteristics were not significantly different between participants and those lost to follow-up with the exception of mental health and depression (Table 1). Specifically, individuals lost to follow-up had significantly lower mental health scores (measured by the SF-12 Mental Component Scale) and significantly higher depression scores (measured by the Zung self-rating depression scale).

At six-month follow-up, 130 patients were classified as having non-persistent LBP with 38 (23\%) clas-

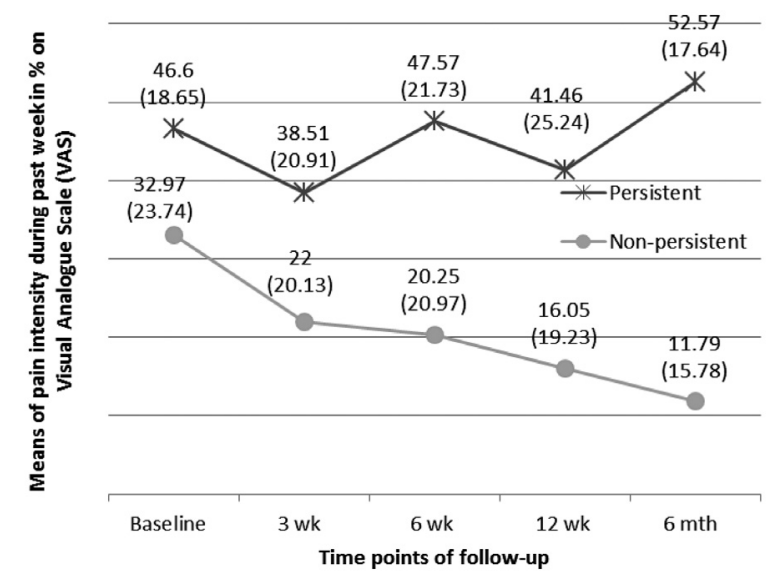

Fig. 1. VAS scores in persistent and non-persistent LBP groups over time. VAS scores are shown as mean (SD).

sified as having persistent LBP. VAS baseline scores in the non-persistent LBP group ranged between 0 and 95 mean $($ mean $=33 \mathrm{~mm})$, and scores in the persistent LBP group between 21 and $88 \mathrm{~mm}($ mean $=47 \mathrm{~mm})$, revealing a significantly higher pain intensity during the past week at baseline for the persistent LBP group $(P<0.001)$.

As shown in Fig. 1, trend analysis revealed clear differences in the sequential reports of pain over time between the non-persistent LBP group and the persistent LBP group, suggesting they are indeed distinct groups of patients. The trend analysis confirmed a decline of the VAS over time for the non-persistent LBP group. This was a first-order trend, with a linear decrease over all five time points measured from baseline to six months from an initial mean VAS score of 33 to a score of 12 at six months. In the persistent LBP group, the trend analysis did not demonstrate a firstorder trend for the VAS scores over time (considering all five time points), confirming that an overall course of non-improvement was present. For this group, VAS scores fluctuated but remained in a range between approximately 40 and 53. Patient characteristics at baseline and at different follow-up time points in Table 2 for the non-persistent LBP group and in Table 3 for the persistent LBP group show in addition to the findings from the regression analyses detailed further below that patients in the non-persistent group attended health practitioners and received treatments and diagnostics less frequently.

\subsection{Occupational domain factors}

Social support at work at baseline correlated inversely with persistent LBP defined by the VAS score 
Table 1

Baseline characteristics of participants who completed six-month follow-up vs. participants lost to follow-up

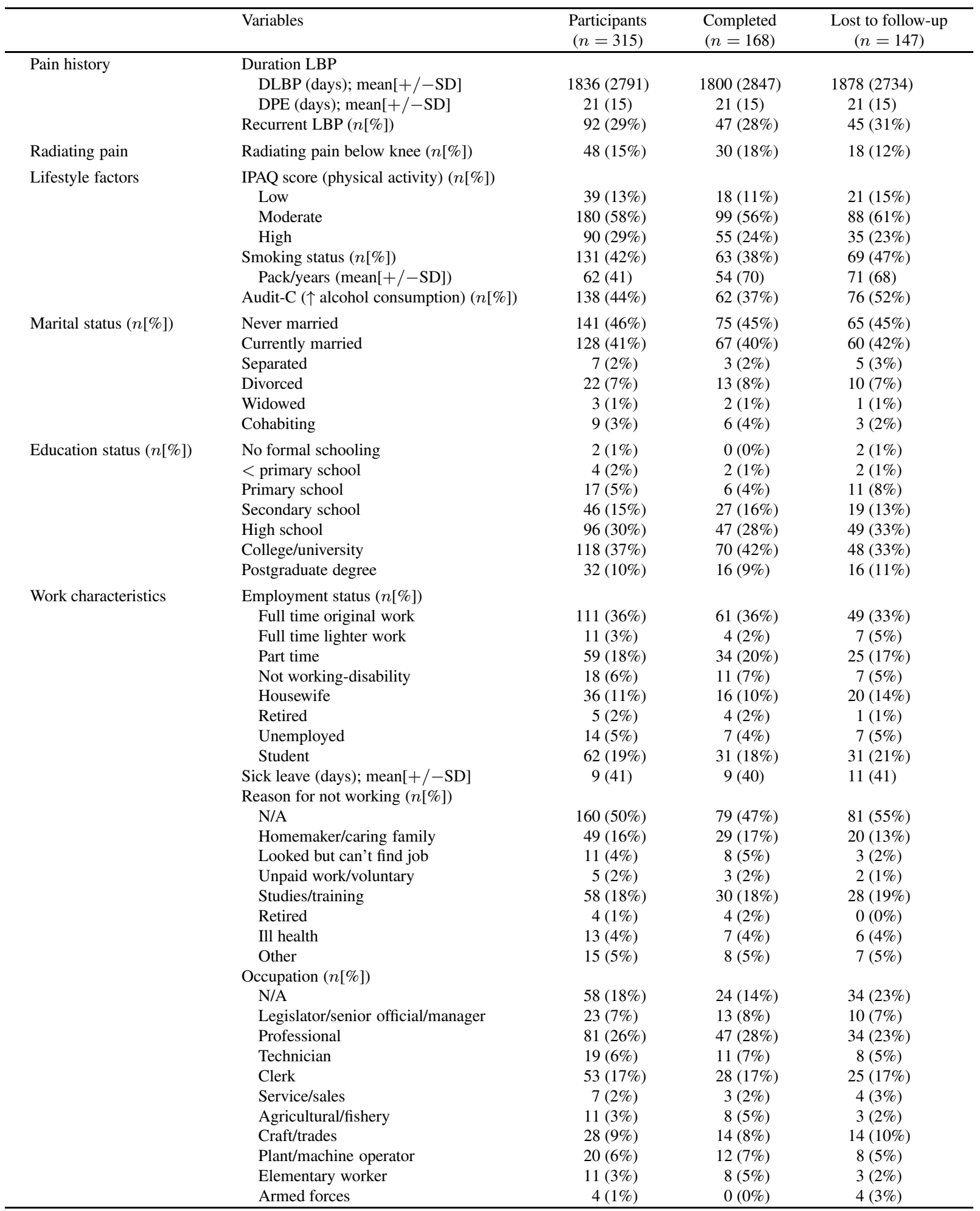


Table 1, continued

\begin{tabular}{|c|c|c|c|c|}
\hline & Variables & $\begin{array}{l}\text { Participants } \\
(n=315)\end{array}$ & $\begin{array}{l}\text { Completed } \\
(n=168)\end{array}$ & $\begin{array}{l}\text { Lost to follow-up } \\
\quad(n=147)\end{array}$ \\
\hline Compensation and benefits & $\begin{array}{l}\text { LBP covered by ACC }(n[\%]) \\
\text { Other benefits }(n[\%]) \\
\text { Health insurance }(n[\%]) \\
\text { Salary replacement }(n[\%]) \\
\text { Healthcare replacement }(n[\%]) \\
\text { Claimed lump sum }(n[\%]) \\
\text { Received lump sum }(n[\%])\end{array}$ & $\begin{array}{r}122(39 \%) \\
47(15 \%) \\
39(13 \%) \\
54(17 \%) \\
51(16 \%) \\
51(16 \%) \\
11(4 \%)\end{array}$ & $\begin{array}{l}65(39 \%) \\
19(9 \%) \\
25(15 \%) \\
32(19 \%) \\
30(18 \%) \\
25(15 \%) \\
6(4 \%)\end{array}$ & $\begin{array}{l}57(40 \%) \\
28(12 \%) \\
14(10 \%) \\
22(15 \%) \\
21(14 \%) \\
26(18 \%) \\
5(3 \%)\end{array}$ \\
\hline Functional limitation & $\begin{array}{l}\text { ODI }(\text { mean }[+/- \text { SD] }) \\
\text { Minimal disability }(0-20)(n[\%]) \\
\text { Moderate disability }(21-40)(n[\%]) \\
\text { Severe disability }(41-60)(n[\%]) \\
\text { Crippled }(>61)(n[\%])\end{array}$ & $\begin{aligned} 22 & (13) \\
168 & (53 \%) \\
119 & (38 \%) \\
27 & (9 \%) \\
1 & (0.3 \%)\end{aligned}$ & $\begin{array}{l}21(13) \\
92(54 \%) \\
63(38 \%) \\
12(7 \%) \\
1(1 \%)\end{array}$ & $\begin{array}{l}23(12) \\
76(52 \%) \\
56(38 \%) \\
15(10 \%) \\
0(0 \%)\end{array}$ \\
\hline General health & $\begin{array}{l}\text { SF-12-PCS (mean }[+/-S D]) \\
\text { SF-12-MCS (mean }[+/-S D])\end{array}$ & $\begin{array}{l}45(9) \\
45(11)\end{array}$ & $\begin{array}{l}45(9) \\
47(10)\end{array}$ & $\begin{array}{l}45(9) \\
43(11)\end{array}$ \\
\hline Pain & $\begin{array}{l}\text { Sensory pain (mean }[+/-\mathrm{SD}]) \\
\text { Affective pain }(\text { mean }[+/-\mathrm{SD}]) \\
\text { Total pain }(\text { mean }[+/-\mathrm{SD}]) \\
\text { Pain intensity last week }(\mathrm{VAS})(\text { mean }[+/-\mathrm{SD}]) \\
\text { Present pain intensity }(n[\%]) \\
\quad \text { No pain } \\
\text { Mild } \\
\text { Discomforting } \\
\text { Distressing } \\
\text { Horrible } \\
\text { Excruciating }\end{array}$ & $\begin{array}{r}28(18) \\
9(13) \\
37(26) \\
37(24) \\
\\
40(13 \%) \\
114(36 \%) \\
131(41 \%) \\
19(6 \%) \\
9(3 \%) \\
2(1 \%)\end{array}$ & $\begin{array}{l}27(18) \\
7(9) \\
34(24) \\
36(24) \\
21(12 \%) \\
62(37 \%) \\
67(40 \%) \\
11(7 \%) \\
6(4 \%) \\
1(1 \%)\end{array}$ & $\begin{array}{l}29(18) \\
11(16) \\
40(27) \\
38(23) \\
19(13 \%) \\
52(36 \%) \\
64(44 \%) \\
8(5 \%) \\
3(2 \%) \\
1(1 \%)\end{array}$ \\
\hline Psychological factors & $\begin{array}{l}\text { DRAM classification (depression/somatization) }(n[\%]) \\
\text { No depression: ZUNG }<17 \\
\text { At risk: ZUNG 17-33; MSPQ }<12 \\
\text { Distressed depressive: ZUNG }>33 \\
\text { Distressed somatic: ZUNG 17-33; } \\
\text { MSPQ >12) } \\
\text { Fear avoidance beliefs (FAB) } \\
\text { Work activity (mean[+/-SD]) } \\
\text { Physical activity (mean[+/-SD]) } \\
\text { FAB total (mean }[+/-\mathrm{SD}]) \\
\text { Catastrophizing (PCS) } \\
\text { Rumination (mean[+/-SD]) } \\
\text { Magnification (mean[+/-SD]) } \\
\text { Helplessness (mean[+/-SD]) } \\
\text { PCS total (mean[+/-SD]) } \\
\text { Non-catastrophizers } \\
\text { Intermediate catastrophizers } \\
\text { Catastrophizers }\end{array}$ & $\begin{array}{c}105(33 \%) \\
97(31 \%) \\
57(18 \%) \\
56(17 \%) \\
13(11) \\
14(6) \\
35(18) \\
5.2(4.5) \\
3.2(3.0) \\
6.2(5.6) \\
14.6(11.8) \\
188(60 \%) \\
64(20 \%) \\
63(20 \%)\end{array}$ & $\begin{array}{c}69(41 \%) \\
48(29 \%) \\
26(16 \%) \\
\\
25(15 \%) \\
\\
13(10) \\
14(6) \\
35(18) \\
4.9(4.3) \\
2.8(2.7) \\
5.8(5.2) \\
13.5(11.2) \\
109(65 \%) \\
29(17 \%) \\
30(18 \%)\end{array}$ & $\begin{array}{c}36(25 \%) \\
49(33 \%) \\
31(21 \%) \\
31(21 \%) \\
\\
13(11) \\
13(6) \\
35(19) \\
\\
5.6(4.6) \\
3.5(3.2) \\
6.8(6.0) \\
15.9(12.4) \\
79(54 \%) \\
35(24 \%) \\
33(22 \%)\end{array}$ \\
\hline Occupational factors & $\begin{array}{l}\text { Job satisfaction (mean }[+/-\mathrm{SD}]) \\
\text { Resigned attitude towards the job (mean }[+/-\mathrm{SD}]) \\
\text { Job content } \\
\text { Method control (mean }[+/-\mathrm{SD}]) \\
\text { Time control (mean }[+/-\mathrm{SD}]) \\
\text { Uncertainty (mean }[+/-\mathrm{SD}]) \\
\text { Work organizational problems (mean }[+/-\mathrm{SD}]) \\
\text { Work interruptions (mean }[+/-\mathrm{SD}]) \\
\text { Concentration requirements }(\text { mean }[+/-\mathrm{SD}]) \\
\text { Time pressure (mean }[+/-\mathrm{SD}]) \\
\text { Single-sided physical stress }(\text { mean }[+/-\mathrm{SD}]) \\
\text { Emotion suppression }(\text { mean }[+/-\mathrm{SD}]) \\
\text { Social support } \\
\text { At home (mean }[+/-\mathrm{SD}]) \\
\text { At work (mean }[+/-\mathrm{SD}]) \\
\text { Total (mean }[+/-\mathrm{SD}])\end{array}$ & $\begin{array}{l}3.7(1.0) \\
3.2(1.5) \\
3.6(1.2) \\
3.3(1.3) \\
2.4(0.9) \\
2.6(0.7) \\
3.0(1.0) \\
3.2(0.9) \\
3.1(1.0) \\
3.0 .(0.7) \\
3.0(1.3)\end{array}$ & $\begin{array}{l}3.7(1.2) \\
3.2(1.3) \\
3.6(1.1) \\
3.2(1.3) \\
2.4(0.8) \\
2.7(0.7) \\
3.1(1.0) \\
3.2(0.9) \\
3.1(0.9) \\
3.1(0.6) \\
2.9(1.2)\end{array}$ & $\begin{array}{l}3.7(1.0) \\
3.2(1.6) \\
3.6(1.2) \\
3.3(1.2) \\
2.4(0.9) \\
2.6(0.7) \\
3.0(1.0) \\
3.1(1.0) \\
3.2(1.1) \\
3.0(0.7) \\
3.0(1.3)\end{array}$ \\
\hline
\end{tabular}


Table 1 , continued

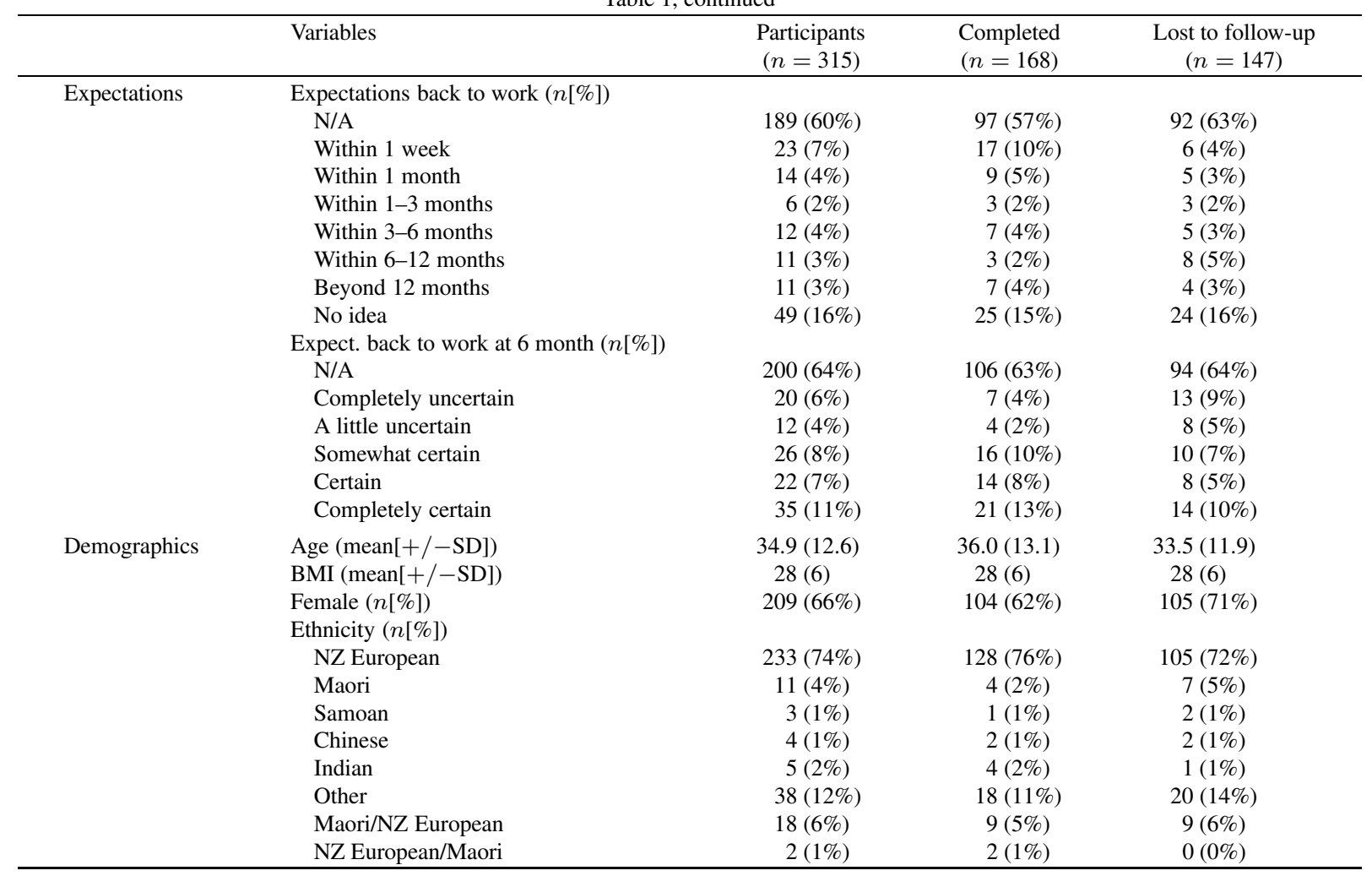

Figures are given as numbers (percentages) or mean (+/-SD) where appropriate; 'low', 'moderate' and 'high' according to IPAQ (International Physical Activity Questionnaire) score; 'smoking status' and 'pack/years' include regular smokers and ex-smokers; pack/years = (packs smoked per day) $\times$ (years as a smoker); 'increased alcohol consumption' is defined in regards to Audit-C.

at six-month follow-up $(r=-0.27 ; p<0.001)$. The odds of having persistent LBP were 0.46 (95\% CI $0.27-$ 0.79; Table 4). A chi squared test of the full occupational predictor model was not significant $\left(\chi^{2}=18.8\right.$, $d f=16, p=0.28)$. Occupational factors and control variables predicted $20 \%$ variance in persistent LBP. The accuracy of the model was $81 \%$ with $98 \%$ of nonpersistent and $23 \%$ of persistent LBP patients correctly identified.

\subsection{Psychological domain factors}

Somatization $(r=0.23 ; p<0.01)$ and depression $(r=0.21 ; p<0.01)$ at baseline correlated with persistent LBP at six-month follow-up. The odds of having persistent LBP were 1.08 for somatization (95\% CI 1.004-1.16; Table 4); multivariate logistic regression was not significant for depression. A chi squared test of the full psychological predictor model was not significant $\left(\chi^{2}=14.8, d f=8, p=0.06\right)$. Psychological factors and control variables predicted $14 \%$ variance in persistent LBP. The accuracy of the model was $80 \%$ with $98 \%$ of non-persistent and $17 \%$ of persistent LBP patients correctly identified.

\subsection{Biomedical domain factors}

Functional limitation $(r=0.16 ; p<0.05)$, sensory pain $(r=0.23 ; p<0.01)$, affective pain $(r=$ $0.24 ; p<0.01)$, pain intensity $(r=0.23 ; p<0.01)$ and duration of $\operatorname{LBP}(r=0.15 ; p<0.05)$ at baseline correlated with persistent LBP at six-month follow-up. Mental well-being correlated inversely with persistent LBP at six-month follow-up $(r=-0.22 ; p<0.01)$. Multivariate logistic regression was not significant for any of these factors.

\subsection{Demographic/lifestyle domain factors}

No significant correlations were found between demographic/lifestyle factors and the presence of persistent LBP at six-month follow-up.

\subsection{Final trimmed model}

When combining only significant variables from the different domains that showed unique predictive power 
Table 2

Characteristics of patients with non-persistent LBP at baseline and different follow-up time points

\begin{tabular}{|c|c|c|c|c|c|c|}
\hline \multicolumn{2}{|l|}{ Variables } & \multirow{2}{*}{$\begin{array}{c}\begin{array}{c}\text { Baseline } \\
(n=130)\end{array} \\
\text { N/A }\end{array}$} & \multirow{2}{*}{$\begin{array}{c}\begin{array}{c}3 w k \text { FU } \\
(n=127)\end{array} \\
1.4(1.9)\end{array}$} & \multirow{2}{*}{$\begin{array}{c}\begin{array}{c}6 w k \text { FU } \\
(n=129)\end{array} \\
1.1(1.7)\end{array}$} & \multirow{2}{*}{$\begin{array}{c}\begin{array}{c}12 \mathrm{wk} \mathrm{FU} \\
(n=126)\end{array} \\
0.8(1.6)\end{array}$} & \multirow{2}{*}{$\begin{array}{c}\begin{array}{c}6 \mathrm{mth} \text { FU } \\
(n=130)\end{array} \\
0.6(1.2)\end{array}$} \\
\hline Days of reduction in nor & rmal activities due to LBP last week (mean[+/-SD]) & & & & & \\
\hline \multirow[t]{8}{*}{ Medication } & $\begin{array}{l}\text { Medication taken last week }(n[\%]) \\
\text { Type of medication }\end{array}$ & N/A & $82(65 \%)$ & $71(55 \%)$ & $68(54 \%)$ & $53(41 \%)$ \\
\hline & N/A & N/A & $45(36 \%)$ & $57(44 \%)$ & $58(46 \%)$ & $77(59 \%)$ \\
\hline & Analgesics & N/A & $31(24 \%)$ & $33(26 \%)$ & $27(21 \%)$ & $21(16 \%)$ \\
\hline & NSAIDS & N/A & $15(12 \%)$ & $12(9 \%)$ & $12(10 \%)$ & $7(5 \%)$ \\
\hline & Strong analgesics & N/A & $3(2 \%)$ & $1(1 \%)$ & $3(2 \%)$ & $1(1 \%)$ \\
\hline & Analgesics/NSAIDS & N/A & $28(22 \%)$ & $20(16 \%)$ & $21(17 \%)$ & $18(14 \%)$ \\
\hline & Analgesics/NSAIDS/strong analgesics & N/A & $2(2 \%)$ & $3(2 \%)$ & $5(4 \%)$ & $5(4 \%)$ \\
\hline & Analgesics/strong analgesics & N/A & $3(2 \%)$ & $2(2 \%)$ & $0(0 \%)$ & $1(1 \%)$ \\
\hline \multirow{5}{*}{$\begin{array}{l}\text { Satisfaction with } \\
\text { condition }(n[\%])\end{array}$} & Very satisfied & N/A & $11(9 \%)$ & $23(18 \%)$ & $22(18 \%)$ & $40(31 \%)$ \\
\hline & Somewhat satisfied & N/A & $21(16 \%)$ & $35(28 \%)$ & $39(31 \%)$ & $42(32 \%)$ \\
\hline & Neither satisfied nor dissatisfied & N/A & $20(16 \%)$ & $21(16 \%)$ & $24(19 \%)$ & $17(13 \%)$ \\
\hline & Somewhat dissatisfied & N/A & $48(38 \%)$ & $32(25 \%)$ & $30(24 \%)$ & $21(16 \%)$ \\
\hline & Very dissatisfied & N/A & $27(21 \%)$ & $17(13 \%)$ & $10(8 \%)$ & $10(8 \%)$ \\
\hline \multirow{5}{*}{$\begin{array}{l}\text { Satisfaction with } \\
\text { medical care }(n[\%])\end{array}$} & Very satisfied & N/A & $27(21 \%)$ & $38(30 \%)$ & $35(28 \%)$ & $40(31 \%)$ \\
\hline & Somewhat satisfied & N/A & $45(37 \%)$ & $45(35 \%)$ & $49(39 \%)$ & $47(37 \%)$ \\
\hline & Neither satisfied nor dissatisfied & N/A & $33(26 \%)$ & $30(23 \%)$ & $27(22 \%)$ & $24(19 \%)$ \\
\hline & Somewhat dissatisfied & N/A & $20(16 \%)$ & $13(10 \%)$ & $12(10 \%)$ & $16(12 \%)$ \\
\hline & Very dissatisfied & N/A & $2(2 \%)$ & $2(2 \%)$ & $1(1 \%)$ & $1(1 \%)$ \\
\hline \multirow[t]{13}{*}{ Return to work $(n[\%])$} & N/A & N/A & $69(55 \%)$ & $59(46 \%)$ & $64(51 \%)$ & $73(56 \%)$ \\
\hline & Back to the same job & N/A & $29(23 \%)$ & $36(28 \%)$ & $27(21 \%)$ & $30(22 \%)$ \\
\hline & Back, but modified & N/A & $8(6 \%)$ & $7(5 \%)$ & $5(4 \%)$ & $2(2 \%)$ \\
\hline & In a new job & N/A & $1(1 \%)$ & $0(0 \%)$ & $1(1 \%)$ & $2(2 \%)$ \\
\hline & Not working because of pain & N/A & $3(2 \%)$ & $2(2 \%)$ & $2(2 \%)$ & $5(4 \%)$ \\
\hline & Not working for other reasons & N/A & $7(6 \%)$ & $1(1 \%)$ & $3(2 \%)$ & $1(1 \%)$ \\
\hline & Homemaker/caring for family & N/A & $5(4 \%)$ & $8(6 \%)$ & $12(10 \%)$ & $8(6 \%)$ \\
\hline & Looked but can't find a job & N/A & $0(0 \%)$ & $2(2 \%)$ & $1(1 \%)$ & $2(2 \%)$ \\
\hline & Doing unpaid work/voluntary & N/A & $1(1 \%)$ & $1(1 \%)$ & $2(2 \%)$ & $2(2 \%)$ \\
\hline & Studies/training & N/A & $3(2 \%)$ & $7(5 \%)$ & $6(5 \%)$ & $3(2 \%)$ \\
\hline & Retired & N/A & $0(0 \%)$ & $0(0 \%)$ & $0(0 \%)$ & $0(0 \%)$ \\
\hline & Ill health & N/A & $0(0 \%)$ & $2(2 \%)$ & $1(1 \%)$ & $1(1 \%)$ \\
\hline & Other & N/A & $0(0 \%)$ & $2(2 \%)$ & $1(1 \%)$ & $0(0 \%)$ \\
\hline \multicolumn{2}{|c|}{ Days of sick leave due to LBP last week (mean[+/-SD]) } & N/A & $0.5(1.4)$ & $0.3(0.9)$ & $0.3(1.2)$ & $0.2(0.9)$ \\
\hline \multirow[t]{5}{*}{ Functional limitation } & ODI (mean $[+/-\mathrm{SD}])$ & $21(13)$ & $18(13)$ & $15(13)$ & $14(14)$ & $13(13)$ \\
\hline & Minimal disability $(0-20)(n[\%])$ & $76(59 \%)$ & $85(67 \%)$ & $91(71 \%)$ & $101(80 \%)$ & $111(85 \%)$ \\
\hline & Moderate disability $(21-40)(n[\%])$ & $46(35 \%)$ & $36(28 \%)$ & $31(24 \%)$ & $16(13 \%)$ & $11(9 \%)$ \\
\hline & Severe disability $(41-60)(n[\%])$ & $7(5 \%)$ & $5(4 \%)$ & $7(5 \%)$ & $8(6 \%)$ & $7(5 \%)$ \\
\hline & Crippled $(>61)(n[\%])$ & $1(1 \%)$ & $1(1 \%)$ & $0(0 \%)$ & $1(1 \%)$ & $1(1 \%)$ \\
\hline \multirow[t]{2}{*}{ General health } & SF-12-PCS (mean[+/-SD]) & $45(9)$ & $47(9)$ & $49(9)$ & $50(8)$ & $51(8)$ \\
\hline & SF-12-MCS (mean $[+/-S D])$ & $48(10)$ & $48(11)$ & $49(10)$ & $49(11)$ & $49(10)$ \\
\hline \multirow[t]{10}{*}{ Pain } & Sensory pain $($ mean $[+/-S D])$ & $25(17)$ & $19(18)$ & $16(17)$ & $14(18)$ & $11(14)$ \\
\hline & Affective pain $($ mean $[+/-S D])$ & $6(8)$ & $12(17)$ & $12(18)$ & $10(18)$ & $6(13)$ \\
\hline & Total pain $($ mean $[+/-\mathrm{SD}])$ & $32(23)$ & $31(31)$ & $28(31)$ & $24(34)$ & $17(25)$ \\
\hline & VAS (pain intensity last week) (mean $[+/-\mathrm{SD}])$ & $33(24)$ & $22(20)$ & $20(21)$ & $16(19)$ & $12(16)$ \\
\hline & No pain & $19(15 \%)$ & $30(24 \%)$ & $38(29 \%)$ & $54(43 \%)$ & $71(54 \%)$ \\
\hline & Mild & $47(36 \%)$ & $53(42 \%)$ & $62(48 \%)$ & $49(39 \%)$ & $40(31 \%)$ \\
\hline & Discomforting & $51(39 \%)$ & $36(28 \%)$ & $24(19 \%)$ & $18(14 \%)$ & $12(9 \%)$ \\
\hline & Distressing & $9(7 \%)$ & $8(6 \%)$ & $4(3 \%)$ & $4(3 \%)$ & $6(5 \%)$ \\
\hline & Horrible & $4(3 \%)$ & $0(0 \%)$ & $1(1 \%)$ & $1(1 \%)$ & $1(1 \%)$ \\
\hline & Excruciating & $0(0 \%)$ & $0(0 \%)$ & $0(0 \%)$ & $0(0 \%)$ & $0(0 \%)$ \\
\hline \multirow[t]{4}{*}{ Psychological factors } & DRAM classification (depression/somatization) $(n[\%])$ & & & & & \\
\hline & No depression: ZUNG $<17$ & $60(46 \%)$ & $63(50 \%)$ & $83(65 \%)$ & $78(62 \%)$ & $82(63 \%)$ \\
\hline & At risk: ZUNG 17-33; MSPQ $<12$ & $37(29 \%)$ & $38(30 \%)$ & $21(16 \%)$ & $28(22 \%)$ & $24(18 \%)$ \\
\hline & Distressed depressive: $\mathrm{ZUNG}>33$ & $16(12 \%)$ & $14(11 \%)$ & $13(10 \%)$ & $10(8 \%)$ & $5(4 \%)$ \\
\hline
\end{tabular}


Table 2, continued

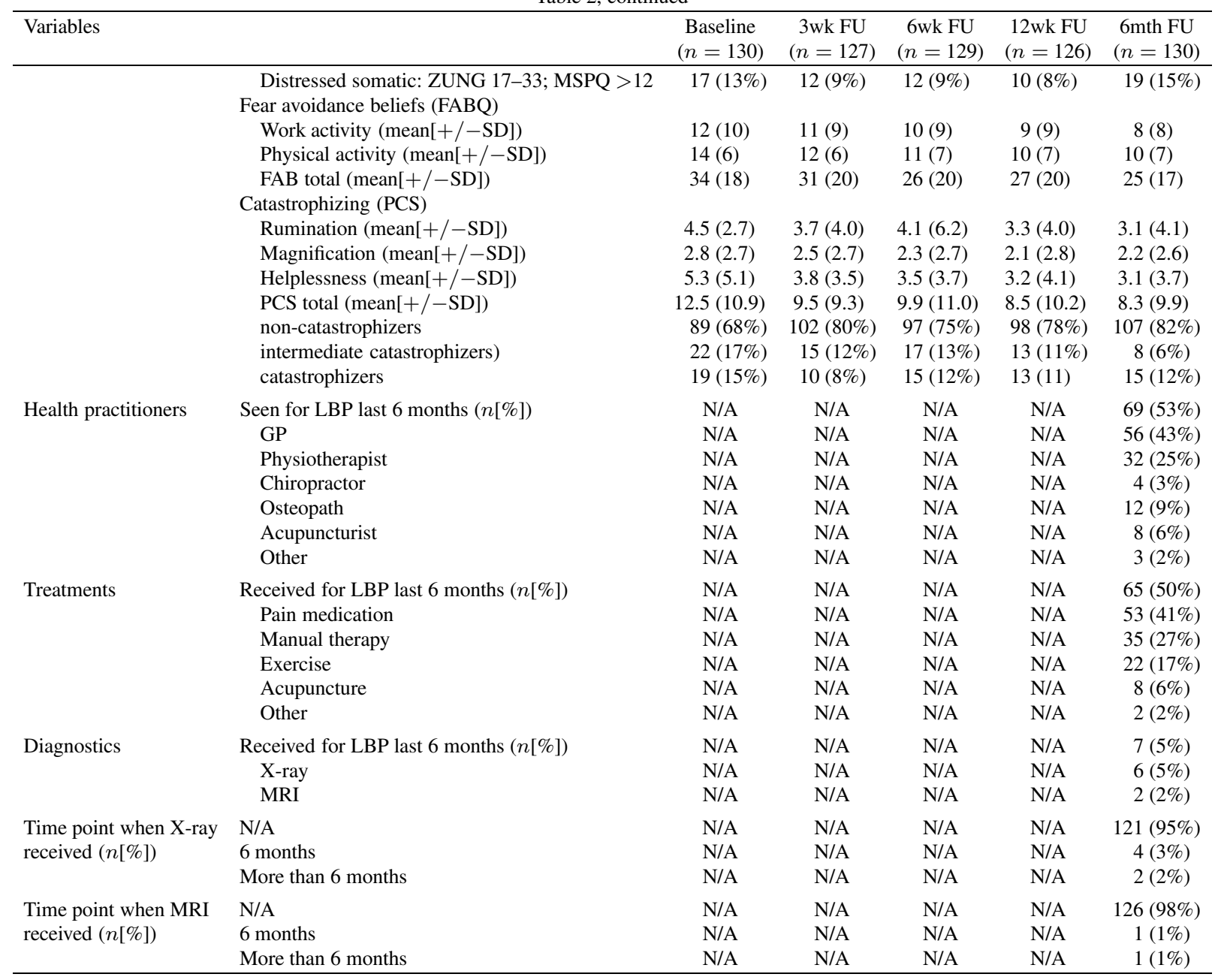

Figures are given as numbers (percentages) or mean $(+/-\mathrm{SD})$ where appropriate; participants in ' 3 wk FU' subgroup with variable 'return to work' do not add up to the total number of participants due to missing data; participants in '6wk FU' subgroup with variables 'medications taken last week, 'type of medications', 'satisfaction with condition' and 'satisfaction with medical care' do not add up to the total number of participants due to missing data; participants in ' $12 \mathrm{wk}$ FU' subgroup with variables 'satisfaction with condition', 'satisfaction with medical care' and 'catastrophizing' do not add up to the total number of participants due to missing data; participants in '6mth FU' subgroup with variables 'satisfaction with medical care', 'health practitioners seen last 6 months', 'treatment received in last 6 months', 'diagnostics received last 6 months', 'time point when X-ray received' and time point when MRI received' do not add up to the total number of participants due to missing data; participants in '6mth FU' subgroup with variables 'health practitioners', 'treatments' and 'diagnostics' allow for multiple answers.

within their domain variables into one model, a final two-predictor model, comprised of social support at work and somatization, was found to be statistically significant $\left(\chi^{2}=13.1, d f=5, p<0.023\right.$; Table 5). All factors and control variables predicted $14 \%$ of the variance in development of persistent LBP. Additionally, the Chi-Squared Hosmer Lemeshow test was not significant indicating that this final predictor model has a good fit for the data. The accuracy of the final model was $78 \%$, with $97 \%$ of non-persistent and $15 \%$ of persistent LBP patients correctly identified.

\section{Discussion}

The present study evaluated occupational, psychological, biomedical and demographic/lifestyle domains as prognostic factors for persistent LBP. According to the aim of the study, it elucidated their role as risk factors for the development of persistent LBP and as resources for preventing persistent LBP six months after an acute/subacute/recurrent episode of LBP in a primary care setting. Indeed, a two-factor predictive model, consisting of social support at work and soma- 
Table 3

Characteristics of patients with persistent LBP at baseline and different follow-up time points

\begin{tabular}{|c|c|c|c|c|c|c|}
\hline \multicolumn{2}{|l|}{ Variables } & $\begin{array}{l}\text { Baseline } \\
(n=38)\end{array}$ & $\begin{array}{l}3 \text { wk FU } \\
(n=38) \\
\end{array}$ & $\begin{array}{l}6 \text { wk FU } \\
(n=37)\end{array}$ & $\begin{array}{l}12 \text { wk FU } \\
(n=38)\end{array}$ & $\begin{array}{l}6 \mathrm{mth} \text { FU } \\
(n=38)\end{array}$ \\
\hline \multicolumn{2}{|c|}{ Days of reduction in normal activities due to LBP last week (mean[+/-SD]) } & N/A & $2.7(1.9)$ & $2.1(2.0)$ & $2.4(2.0)$ & $2.5(2.3)$ \\
\hline \multirow[t]{9}{*}{ Medication } & $\begin{array}{l}\text { Medication taken last week }(n[\%]) \\
\text { Type of medication }\end{array}$ & N/A & $32(84 \%)$ & $28(76 \%)$ & $30(79 \%)$ & $32(84 \%)$ \\
\hline & N/A & N/A & $6(16 \%)$ & $9(24 \%)$ & $7(21 \%)$ & $8(21 \%)$ \\
\hline & Analgesics & N/A & $14(34 \%)$ & $9(24 \%)$ & $11(29 \%)$ & $7(18 \%)$ \\
\hline & NSAIDS & N/A & $4(11 \%)$ & $6(16 \%)$ & $3(8 \%)$ & $6(16 \%)$ \\
\hline & Strong analgesics & N/A & $0(0 \%)$ & $1(3 \%)$ & $1(3 \%)$ & $4(11 \%)$ \\
\hline & Analgesics/NSAIDS & N/A & $14(37 \%)$ & $7(19 \%)$ & $12(32 \%)$ & $6(16 \%)$ \\
\hline & Analgesics/NSAIDS/strong analgesics & N/A & $0(0 \%)$ & $4(11 \%)$ & $3(8 \%)$ & $2(5 \%)$ \\
\hline & Analgesics/strong analgesics & N/A & $1(3 \%)$ & $1(3 \%)$ & $0(0 \%)$ & $3(8 \%)$ \\
\hline & NSAIDS/strong analgesics & N/A & $0(0 \%)$ & $0(0 \%)$ & $0(0 \%)$ & $1(3 \%)$ \\
\hline \multirow{5}{*}{$\begin{array}{l}\text { Satisfaction with } \\
\text { condition }(n[\%])\end{array}$} & Very satisfied & N/A & $0(0 \%)$ & $2(5 \%)$ & $1(3 \%)$ & $2(5 \%)$ \\
\hline & Somewhat satisfied & N/A & $2(5 \%)$ & $2(5 \%)$ & $6(15 \%)$ & $3(8 \%)$ \\
\hline & Neither satisfied nor dissatisfied & N/A & $2(5 \%)$ & $4(11 \%)$ & $6(15 \%)$ & $4(11 \%)$ \\
\hline & Somewhat dissatisfied & N/A & $23(61 \%)$ & $16(43 \%)$ & $13(34 \%)$ & $14(37 \%)$ \\
\hline & Very dissatisfied & N/A & $11(29 \%)$ & $13(35 \%)$ & $12(32 \%)$ & $15(40 \%)$ \\
\hline \multirow{5}{*}{$\begin{array}{l}\text { Satisfaction with } \\
\text { medical care }(n[\%])\end{array}$} & Very satisfied & N/A & $4(11 \%)$ & $3(8 \%)$ & $5(13 \%)$ & $4(11 \%)$ \\
\hline & Somewhat satisfied & N/A & $12(32 \%)$ & $12(33 \%)$ & $12(32 \%)$ & $11(29 \%)$ \\
\hline & Neither satisfied nor dissatisfied & N/A & $10(27 \%)$ & $13(36 \%)$ & $9(23 \%)$ & $8(21 \%)$ \\
\hline & Somewhat dissatisfied & N/A & $6(16 \%)$ & $8(22 \%)$ & $9(23 \%)$ & $9(23 \%)$ \\
\hline & Very dissatisfied & N/A & $5(14 \%)$ & $0(0 \%)$ & $3(8 \%)$ & $6(16 \%)$ \\
\hline \multirow[t]{13}{*}{ Return to work $(n[\%])$} & N/A & N/A & $19(50 \%)$ & $22(60 \%)$ & $21(55 \%)$ & $18(47 \%)$ \\
\hline & Back to the same job & N/A & $5(13 \%)$ & $3(8 \%)$ & $3(8 \%)$ & $3(8 \%)$ \\
\hline & Back, but modified & N/A & $0(0 \%)$ & $1(3 \%)$ & $0(0 \%)$ & $0(0 \%)$ \\
\hline & In a new job & N/A & $1(3 \%)$ & $1(3 \%)$ & $1(3 \%)$ & $1(3 \%)$ \\
\hline & Not working because of pain & N/A & $2(5 \%)$ & $3(8 \%)$ & $5(13 \%)$ & $7(18 \%)$ \\
\hline & Not working for other reasons & N/A & $4(11 \%)$ & $2(5 \%)$ & $2(5 \%)$ & $1(3 \%)$ \\
\hline & Homemaker/caring for family & N/A & $2(5 \%)$ & $3(8 \%)$ & $2(5 \%)$ & $4(11 \%)$ \\
\hline & Looked but canâĂŹt find a job & N/A & $1(3 \%)$ & $0(0 \%)$ & $0(0 \%)$ & $1(3 \%)$ \\
\hline & Doing unpaid work/ voluntary & N/A & $0(0 \%)$ & $0(0 \%)$ & $0(0 \%)$ & $1(3 \%)$ \\
\hline & Studies/training & N/A & $0(0 \%)$ & $0(0 \%)$ & $2(5 \%)$ & $2(5 \%)$ \\
\hline & Retired & N/A & $2(5 \%)$ & $1(3 \%)$ & $1(3 \%)$ & $0(0 \%)$ \\
\hline & Ill health & N/A & $2(5 \%)$ & $1(3 \%)$ & $1(3 \%)$ & $0(0 \%)$ \\
\hline & Other & N/A & $0(0 \%)$ & $0(0 \%)$ & $0(0 \%)$ & $0(0 \%)$ \\
\hline \multicolumn{2}{|c|}{ Days of sick leave due to LBP last week (mean $[+/-\mathrm{SD}])$} & N/A & $1.1(2.0)$ & $1.0(2.0)$ & $0.5(1.5)$ & $1.2(2.2)$ \\
\hline \multirow{5}{*}{ Functional limitation } & ODI $($ mean $[+/-$ SD] $)$ & $25(14)$ & $27(16)$ & $28(17)$ & $26(16)$ & $29(15)$ \\
\hline & Minimal disability $(0-20)(n[\%])$ & $15(39 \%)$ & $16(42 \%)$ & $13(35 \%)$ & $15(42 \%)$ & $16(34 \%)$ \\
\hline & Moderate disability $(21-40)(n[\%])$ & $18(47 \%)$ & $14(36 \%)$ & $17(46 \%)$ & $15(39 \%)$ & $16(42 \%)$ \\
\hline & Severe disability $(41-60)(n[\%])$ & $5(13 \%)$ & $7(18 \%)$ & $7(19 \%)$ & $5(13 \%)$ & $8(21 \%)$ \\
\hline & Crippled $(>61)(n[\%])$ & $0(0 \%)$ & $1(3 \%)$ & $1(3 \%)$ & $2(5 \%)$ & $1(3 \%)$ \\
\hline \multirow[t]{2}{*}{ General health } & SF-12-PCS (mean $[+/-\mathrm{SD}])$ & $43(8)$ & $43(9)$ & $43(9)$ & $44(11)$ & $42(10)$ \\
\hline & SF-12-MCS (mean $[+/-S D])$ & $43(9)$ & $44(9)$ & $41(10)$ & $43(10)$ & $43(11)$ \\
\hline \multirow[t]{11}{*}{ Pain } & Sensory pain $($ mean $[+/-\mathrm{SD}])$ & $34(21)$ & $30(19)$ & $31(22)$ & $26(21)$ & $35(23)$ \\
\hline & Affective pain $($ mean $[+/-S D])$ & $11(11)$ & $19(23)$ & $23(22)$ & $21(20)$ & $26(25)$ \\
\hline & Total pain $($ mean $[+/-\mathrm{SD}])$ & $45(29)$ & $49(38)$ & $55(41)$ & $47(39)$ & $61(45)$ \\
\hline & VAS (pain intensity last week) (mean $[+/-\mathrm{SD}])$ & $46(18)$ & $39(21)$ & $48(22)$ & $43(25)$ & $54(18)$ \\
\hline & Present pain intensity $(n[\%])$ & & & & & \\
\hline & No pain & $2(5 \%)$ & $1(3 \%)$ & $3(8 \%)$ & $8(21 \%)$ & $3(8 \%)$ \\
\hline & Mild & $12(31 \%)$ & $13(34 \%)$ & $13(35 \%)$ & $9(24 \%)$ & $8(21 \%)$ \\
\hline & Discomforting & $19(50 \%)$ & $18(47 \%)$ & $15(41 \%)$ & $13(34 \%)$ & $16(42 \%)$ \\
\hline & Distressing & $2(5 \%)$ & $4(11 \%)$ & $4(11 \%)$ & $8(16 \%)$ & $7(18 \%)$ \\
\hline & Horrible & $2(5 \%)$ & $2(5 \%)$ & $2(4 \%)$ & $2(5 \%)$ & $3(8 \%)$ \\
\hline & Excruciating & $1(3 \%)$ & $0(0 \%)$ & $0(0 \%)$ & $0(0 \%)$ & $1(3 \%)$ \\
\hline \multirow[t]{3}{*}{ Psychological factors } & DRAM classification (depression/somatization) $(n[\%])$ & & & & & \\
\hline & No depression: ZUNG $<17$ & $9(23 \%)$ & $8(21 \%)$ & $7(19 \%)$ & $17(46 \%)$ & $9(23 \%)$ \\
\hline & At risk: ZUNG 17-33; MSPQ $<12$ & $10(26 \%)$ & $14(37 \%)$ & $11(30 \%)$ & $5(11 \%)$ & $10(26 \%)$ \\
\hline
\end{tabular}


Table 3, continued

\begin{tabular}{|c|c|c|c|c|c|c|}
\hline \multicolumn{2}{|l|}{ Variables } & \multirow{2}{*}{$\begin{array}{c}\begin{array}{c}\text { Baseline } \\
(n=38)\end{array} \\
10(26 \%)\end{array}$} & \multirow{2}{*}{$\begin{array}{r}\begin{array}{c}3 \text { wk FU } \\
(n=38)\end{array} \\
7(18 \%)\end{array}$} & \multirow{2}{*}{$\begin{array}{c}\begin{array}{c}6 w k \mathrm{FU} \\
(n=37)\end{array} \\
11(30 \%)\end{array}$} & \multirow{2}{*}{$\begin{array}{r}\begin{array}{r}12 w k \text { FU } \\
(n=38)\end{array} \\
6(16 \%)\end{array}$} & \multirow{2}{*}{$\begin{array}{c}\begin{array}{c}6 \mathrm{mth} \text { FU } \\
(n=38)\end{array} \\
10(26 \%)\end{array}$} \\
\hline & Distressed depressive: $\mathrm{ZUNG}>33$ & & & & & \\
\hline & Distressed somatic: ZUNG 17-33; MSPQ >12 & $9(23 \%)$ & $9(24 \%)$ & $8(22 \%)$ & $11(29 \%)$ & $9(23 \%)$ \\
\hline & Fear Avoidance beliefs (FABQ) & & & & & \\
\hline & Work activity $($ mean $[+/-\mathrm{SD}])$ & $14(11)$ & $13(10)$ & $14(11)$ & $12(9)$ & $13(12)$ \\
\hline & Physical activity (mean $[+/-\mathrm{SD}])$ & $16(6)$ & $15(5)$ & $14(7)$ & $12(9)$ & $14(12)$ \\
\hline & FAB total $($ mean $[+/-\mathrm{SD}])$ & $37(20)$ & 39 (19) & $38(24)$ & $35(22)$ & $39(24)$ \\
\hline & Catastrophizing (PCS) & & & & & \\
\hline & Rumination (mean $[+/-\mathrm{SD}])$ & $6.2(4.9)$ & $5.8(4.5)$ & $6.1(5.7)$ & $5.8(5.2)$ & $6.1(5.4)$ \\
\hline & Magnification $(\operatorname{mean}[+/-\mathrm{SD}])$ & $3.0(2.7)$ & $3.6(3.1)$ & $3.8(3.6)$ & $3.0(2.9)$ & $3.5(3.6)$ \\
\hline & Helplessness (mean $[+/-\mathrm{SD}])$ & $7.0(5.5)$ & $5.9(4.3)$ & $6.5(5.4)$ & $6.0(5.5)$ & $6.5(5.5)$ \\
\hline & PCS total (mean $[+/-\mathrm{SD}])$ & $16.3(11.8)$ & $15.2(10.1)$ & $16.4(14.0)$ & $14.6(12.8)$ & $16.3(13.6)$ \\
\hline & Non-catastrophizers & $20(53 \%)$ & $18(47 \%)$ & $21(57 \%)$ & $22(60 \%)$ & $22(58 \%)$ \\
\hline & Intermediate catastrophizers) & $9(24 \%)$ & $14(37 \%)$ & $6(16 \%)$ & $4(11 \%)$ & $8(21 \%)$ \\
\hline & Catastrophizers & $9(24 \%)$ & $8(16 \%)$ & $10(27 \%)$ & $11(30 \%)$ & $8(21 \%)$ \\
\hline \multirow{7}{*}{ Health practitioners } & Seen for LBP last 6 months $(n[\%])$ & N/A & N/A & N/A & N/A & $29(78 \%)$ \\
\hline & GP & N/A & N/A & N/A & N/A & $22(61 \%)$ \\
\hline & Physiotherapist & N/A & N/A & N/A & N/A & $15(42 \%)$ \\
\hline & Chiropractor & N/A & N/A & N/A & N/A & $3(8 \%)$ \\
\hline & Osteopath & N/A & N/A & N/A & N/A & $4(11 \%)$ \\
\hline & Acupuncturist & N/A & N/A & N/A & N/A & $1(3 \%)$ \\
\hline & Other & N/A & N/A & N/A & N/A & $2(6 \%)$ \\
\hline \multirow[t]{6}{*}{ Treatments } & Received for LBP last 6 months ( $n[\%])$ & N/A & N/A & N/A & N/A & $28(76 \%)$ \\
\hline & Pain medication & N/A & N/A & N/A & N/A & $24(67 \%)$ \\
\hline & Manual therapy & N/A & N/A & N/A & N/A & $12(33 \%)$ \\
\hline & Exercise & N/A & N/A & N/A & N/A & $9(25 \%)$ \\
\hline & Acupuncture & N/A & N/A & N/A & N/A & $1(3 \%)$ \\
\hline & Other & N/A & N/A & N/A & N/A & $1(3 \%)$ \\
\hline \multirow[t]{3}{*}{ Diagnostics } & Received for LBP last 6 months ( $n[\%])$ & N/A & N/A & N/A & N/A & $5(14 \%)$ \\
\hline & X-ray & N/A & N/A & N/A & N/A & $4(11 \%)$ \\
\hline & MRI & N/A & N/A & N/A & N/A & $1(3 \%)$ \\
\hline \multirow{3}{*}{$\begin{array}{l}\text { Time point when X-ray } \\
\text { received }(n[\%])\end{array}$} & N/A & N/A & N/A & N/A & N/A & $31(86 \%)$ \\
\hline & 6 months & N/A & N/A & N/A & N/A & $1(3 \%)$ \\
\hline & More than 6 months & N/A & N/A & N/A & N/A & $4(11 \%)$ \\
\hline \multirow{3}{*}{$\begin{array}{l}\text { Time point when MRI } \\
\text { received }(n[\%])\end{array}$} & N/A & N/A & N/A & N/A & N/A & $35(97 \%)$ \\
\hline & 6 months & N/A & N/A & N/A & N/A & $0(0 \%)$ \\
\hline & More than 6 months & N/A & N/A & N/A & N/A & $1(3 \%)$ \\
\hline
\end{tabular}

Figures are given as numbers (percentages) or mean $(+/-\mathrm{SD})$ where appropriate; participants in '3wk FU' subgroup with variable 'return to work' do not add up to the total number of participants due to missing data; participants in '6wk FU' subgroup with variables 'medications taken last week, 'type of medications', 'satisfaction with condition' and 'satisfaction with medical care' do not add up to the total number of participants due to missing data; participants in ' 12 wk FU' subgroup with variables 'satisfaction with condition', 'satisfaction with medical care' and 'catastrophizing' do not add up to the total number of participants due to missing data; participants in ' 6 mth FU' subgroup with variables 'satisfaction with medical care', 'health practitioners seen last 6 months', 'treatment received in last 6 months', 'diagnostics received last 6 months', 'time point when X-ray received' and time point when MRI received' do not add up to the total number of participants due to missing data; participants in '6mth FU' subgroup with variables 'health practitioners', 'treatments' and 'diagnostics' allow for multiple answers.

tization, was found to be predictive of the development of persistent LBP at six months. By this the present study addressed a gap of knowledge in the current literature where studies that evaluate both risk factors (for the development of persistent LBP) and protective factors (preventing the development of persistent LBP) in the same cohort are scarce. The four follow-ups of the study confirm persistent pain to be persistent across repeated measurements while much research only relies on post-hoc pain judgements that cover six months.

Social support at work was found to be an occupational protective factor for the development of persis- tent LBP at six-month follow-up. Thus, the study confirms recent evidence for social support as a protective factor $[24,25]$. Social support at work comprises emotional support and assistance by both colleagues and supervisors [26]. This study does not distinguish between different sources of social support at work, i.e. between the social support at work provided by colleagues or supervisors. A future study should differentiate between support providers as findings by Elfering et al. [27] suggest that provider-specific constellations of social support at work may have either a positive or a negative influence on the development of LBP. In ad- 
Table 4

Prognostic variables for persistent LBP in multivariate logistic regression analysis

\begin{tabular}{|c|c|c|c|c|c|c|}
\hline & $B$ & $S E$ & Wald & $p$ & OR & $\mathrm{CI}(\mathrm{OR})$ \\
\hline \multicolumn{7}{|l|}{ Occupational factors at baseline } \\
\hline Social support at work & -0.77 & 0.28 & 7.94 & 0.005 & 0.46 & $0.27-0.79$ \\
\hline Social support at home & 0.34 & 0.25 & 1.88 & 0.170 & 1.41 & $0.86-2.28$ \\
\hline Job control & -0.10 & 0.26 & 0.16 & 0.693 & 0.90 & $0.55-1.50$ \\
\hline Job satisfaction & 0.14 & 0.22 & 0.40 & 0.525 & 1.15 & $0.75-1.78$ \\
\hline Work absenteeism & 0.00 & 0.01 & 0.57 & 0.450 & 1.00 & $0.99-1.01$ \\
\hline Resigned attitude towards the job & 0.39 & 0.20 & 3.74 & 0.053 & 1.48 & $0.99-2.20$ \\
\hline Uncertainty & -0.08 & 0.35 & 0.05 & 0.819 & 0.92 & $0.47-1.83$ \\
\hline Work organizational problems & -0.46 & 0.39 & 1.39 & 0.239 & 0.63 & $0.30-1.34$ \\
\hline Work interruptions & -0.15 & 0.31 & 0.22 & 0.637 & 0.87 & $0.47-1.58$ \\
\hline Concentration requirements & 0.64 & 0.35 & 3.31 & 0.069 & 1.84 & $0.95-3.77$ \\
\hline Time pressure & -0.27 & 0.34 & 0.65 & 0.420 & 0.76 & $0.40-1.47$ \\
\hline Single-sided physical stress & 0.03 & 0.45 & 0.01 & 0.944 & 1.03 & $0.43-2.51$ \\
\hline Emotion suppression & 0.08 & 0.24 & 0.11 & 0.738 & 1.09 & $0.67-1.75$ \\
\hline \multicolumn{7}{|l|}{$\mathrm{R}^{2}=0.199$ (Nagelkerke) } \\
\hline \multicolumn{7}{|l|}{ Model $\chi^{2}=18.8, p=0.28, d f=16$} \\
\hline \multicolumn{7}{|l|}{ Psychological factors at baseline } \\
\hline Somatization & 0.08 & 0.04 & 4.34 & 0.037 & 1.08 & $1.004-1.16$ \\
\hline Depression & 0.03 & 0.02 & 1.51 & 0.219 & 1.03 & $0.98-1.08$ \\
\hline Fear-avoidance (work activity) & 0.01 & 0.02 & 0.09 & 0.769 & 1.01 & $0.97-1.05$ \\
\hline Fear-avoidance (physical activity) & 0.02 & 0.04 & 0.35 & 0.557 & 1.02 & $0.95-1.11$ \\
\hline Catastrophizing & -0.01 & 0.02 & 0.11 & 0.739 & 0.99 & $0.95-1.04$ \\
\hline \multicolumn{7}{|l|}{$\mathrm{R}^{2}=0.136$ (Nagelkerke) } \\
\hline \multicolumn{7}{|l|}{ Model $\chi^{2}=14.8, p=0.06, d f=8$} \\
\hline \multicolumn{7}{|l|}{ Biomedical factors at baseline } \\
\hline Duration of LBP & 0.01 & 0.01 & 2.23 & 0.136 & 1.01 & $0.99-1.03$ \\
\hline Duration of current LBP episode & 0.00 & 0.02 & 0.00 & 0.993 & 1.00 & $0.97-1.03$ \\
\hline Radiating pain & 0.01 & 0.56 & 0.00 & 0.981 & 1.01 & $0.34-3.05$ \\
\hline Functional limitation (ODI) & 0.00 & 0.03 & 0.02 & 0.885 & 0.99 & $0.94-1.05$ \\
\hline Physical health & -0.01 & 0.04 & 0.03 & 0.855 & 0.99 & $0.92-1.07$ \\
\hline Mental health & -0.02 & 0.02 & 0.57 & 0.452 & 0.98 & $0.94-1.03$ \\
\hline Sensory pain & 0.02 & 0.02 & 1.07 & 0.301 & 1.02 & $0.98-1.06$ \\
\hline Affective pain & 0.01 & 0.03 & 0.17 & 0.682 & 1.01 & $0.95-1.08$ \\
\hline Pain intensity last week (VAS) & 0.02 & 0.01 & 1.88 & 0.171 & 1.02 & $0.99-1.04$ \\
\hline \multicolumn{7}{|l|}{$\mathrm{R}^{2}=0.191$ (Nagelkerke) } \\
\hline \multicolumn{7}{|l|}{ Model $\chi^{2}=20.9, p=0.05, d f=12$} \\
\hline \multicolumn{7}{|l|}{ Demographic/lifestyle factors at baseline } \\
\hline Age & 0.02 & 0.03 & 0.40 & 0.527 & 1.02 & $0.97-1.07$ \\
\hline Body mass index & 0.01 & 0.06 & 0.01 & 0.927 & 1.01 & $0.90-1.12$ \\
\hline Gender & -0.21 & 0.77 & 0.08 & 0.780 & 0.81 & $0.18-3.63$ \\
\hline Low physical activity & 0.12 & 1.10 & 0.01 & 0.911 & 1.13 & $0.13-9.68$ \\
\hline Moderate physical activity & -0.59 & 0.80 & 0.55 & 0.460 & 0.55 & $0.12-2.66$ \\
\hline Pack years & 0.01 & 0.01 & 1.88 & 0.170 & 1.01 & $0.99-1.02$ \\
\hline \multicolumn{7}{|l|}{$\mathrm{R}^{2}=0.086$ (Nagelkerke) } \\
\hline Model $\chi^{2}=2.9, p=0.82, d f=$ & & & & & & \\
\hline
\end{tabular}

Criterion: Results are controlled for age, gender and body mass index; $B=$ logistic regression coefficient; $S E=$ standard error; Wald $=$ logistic regression coefficient divided by $S E$, squared; $p=$ significance level of Wald; OR = odds ratio; CI $(\mathrm{OR})=95 \%$ confidence interval of odds ratio; $d f=$ degree of freedom.

dition to a direct protective effect on the development of persistent LBP indicated by the present study, social support at work may also have a moderating effect, with its absence leading to a stronger correlation between a risk factor such as depression and development of persistent LBP [26]. In contrast to results from previous studies [6-8], various occupational risk and protective factors from Hypothesis 1 were not found to be significant in the present study when they were in- cluded together with other domain predictor variables. Thus, many various occupational risk and protective factors showed no unique predictive value within their domain. The domain-based analytic strategy resulted in the most parsimonious predictor model, notably not the best model that can be reached when a more liberal analytical strategy is used.

Our findings that indicate somatization to be a risk factor for the development of persistent LBP have to 
Table 5

Final logistic regression model

\begin{tabular}{lccccc}
\hline Predictors at baseline & $B$ & $S E$ & Wald & $p$ & OR \\
\hline Social support at work & -0.40 & 0.20 & 4.02 & 0.045 & 0.67 \\
Somatization & 0.08 & 0.03 & 5.26 & 0.022 & 1.08 \\
$\quad \mathrm{R}^{2}=13.7$ (Nagelkerke) & & & & \\
$\quad$ Model $\chi^{2}=13.1^{*}, d f=5$ & & & & \\
$\quad$ Sensitivity $=15.1$, Specificity $=97.1$ & & & & & \\
\hline
\end{tabular}

Criterion: Results are controlled for age, gender and body mass index; $B=$ logistic regression coefficient; $S E=$ standard error; Wald $=$ logistic regression coefficient divided by $S E$, squared; $p=$ significance level of Wald; OR = odds ratio; $\mathrm{CI}(\mathrm{OR})=95 \%$ confidence interval of odds ratio; $d f=$ degree of freedom; ${ }^{*}=P<0.05$; two-tailed.

be considered carefully when drawing any conclusions due to the lower limit of the $95 \%$ confidence interval of this factor being 1.004. However, a systematic review on psychological factors as predictors of persistent LBP supports our findings by emphasizing the significance of somatisation and depression on the development of persistent LBP [16]. Furthermore, our findings are in line with results from a recent inception cohort study on prognostic factors in first-time care seekers with acute LBP [28] that showed high baseline scores on a psychosocial screening tool to be significantly associated with non-recovery at twelve months.

Despite some significant bivariate correlations with the development of persistent LBP, no biomedical factors were found to be significant predictors of persistent LBP in multivariate regression. Previous studies have identified various features of pain to be predictive of the development of persistent LBP such as the baseline intensity of pain [17] as well as the location of pain (below the knee) [5]. In the present cohort, baseline pain intensity was similar between groups and only a small number of participants had pain below the knee $(18 \%)$ thus it is not surprising that these variables were not significant predictors in multivariate analysis. Baseline pain levels in the present study were relatively low compared to some other cohorts, therefore, pain may only be a predictor for developing persistent LBP when pain is moderate or greater.

It is of interest that no demographic/lifestyle factors were predictive of the presence of persistent LBP. This is in contrast to a previous systematic review on the relationship between body mass index and development of persistent LBP where it was found that $32 \%$ of all included studies demonstrated a significant correlation between body mass index and the development of persistent LBP [13]. Further, another study indicated a protective effect of physical activity for the development of persistent LBP [19], which was not shown in our study.

This study's final two-predictor model comprising somatization as a predictor for the development of per- sistent LBP and social support at work as a predictor for preventing the development of persistent LBP should be interpreted cautiously. This two predictor parsimonious predictor model explained $14 \%$ of variance of the development of persistent LBP, which is moderate. Although the study covered a broad range of risk and resource factors from various domains, some important factors of unique predictive value may have been missed. For instance, biomechanical work load and genetic factors might improve prediction. Within the predictor model social support at work as a protective resource factor did well identify patients with a low risk of developing persistent LBP (specificity 0.97), whereas somatization as a risk factor did poorly detect patients with a high risk of developing persistent LBP (sensitivity 0.16) [29].

It is also of interest that the predictive factors for the development of persistent pain are different to the predictive factors for the development of persistent disability following an acute/subacute episode of LBP.

One of the strengths of the present study is that it included only validated and commonly-used measures. By implementing measures recommended by the MMICS Statement, this facilitates the comparison of our results with future studies following the same recommendations. A further strength of the present study is that baseline characteristics of participants and individuals lost to follow-up did not show significant differences, except for a lower mental health and a higher depression score for those individuals lost to follow-up. This is typical for study populations where the healthier individuals stay in the study and have even contributed to variance restriction in the risk factor somatization and subsequent underestimation of predictive value.

\subsection{Implications for practice}

Findings from this study confirm the necessity of including prognostic occupational and psychological factors in screening tools for patients at risk of devel- 
oping persistent LBP. Early identification of these patients may facilitate the provision of necessary treatment to reduce the societal and financial burden of persistent LBP as well as avoid the personal burden of persistent pain. Modifiable risk and protective factors should be distinguished from non-modifiable factors to optimise the treatment effect. According to Johanning [30] prevention strategies are an important part of an integrated disability management involving patients with LBP, primary care providers and employers. The benefit of including modifiable protective factors such as social support at work and modifiable risk factors such as somatization in screening tools is that these factors can be addressed in primary and secondary prevention, e.g. in workplace intervention. Feuerstein et al. [31] suggested a secondary prevention strategy directed at improving the work climate through training of supervisors. This could increase the level of social support at work and consequently, reduce the burden of persistent LBP on both the worker and the employer. Future studies that evaluate the effect of modifying these protective and risk factors on the development of persistent LBP are needed.

\section{Conclusion}

In this study of patients with acute/subacute/recurrent LBP from a primary care setting, social support at work was found to be a protective factor against the development of persistent LBP at six months whereas somatization correlated with the progression to persistent LBP. Further research is required to investigate whether different types of social support at work have different prognostic influence on the prevention or development of persistent LBP. Social support at work should be considered as a resource preventing the development of persistent LBP whereas somatization should be considered in workplace interventions for patients suffering from acute, subacute or recurrent LBP.

\section{Acknowledgements}

This work was supported by Lottery Health Research [LHR264307, LHR278283]; the Wishbone Trust [reference 03/09, reference 08/10]; the Bruce McMillan Trust [reference 11/08]; the Dunedin School of Medicine [reference 08/08]; realHealth International [reference 05/08]; and the University of Berne [refer- ence 2009]. MM is funded by a scholarship awarded by the University of Otago. TRS received funding through a Canadian Institutes of Health Research Postdoctoral Training Fellowship. We gratefully acknowledge Kirsten Stout from the Centre for Musculoskeletal Outcomes Research (CMOR) at Dunedin School of Medicine, University of Otago for developing and maintaining the data management system.

\section{References}

[1] da Costa BR, Vieira ER. Risk factors for work-related musculoskeletal disorders: a systematic review of recent longitudinal studies. Am J Ind Med. 2010 Mar; 53(3): 285-323.

[2] Katz JN. Lumbar disc disorders and low-back pain: socioeconomic factors and consequences. J Bone Joint Surg Am. 2006; 88( Suppl 2): 21-24.

[3] Hilfiker R, Bachmann LM, Heitz CAM, Lorenz T, Joronen H, Klipstein A. Value of predictive instruments to determine persisting restriction of function in patients with subacute nonspecific low back pain. Systematic review. Eur Spine J. 2007; 16(11): 1755-1775.

[4] Heneweer H, Aufdemkampe G, van Tulder MW, Kiers H, Stappaerts KH, Vanhees L. Psychosocial variables in patients with (sub)acute low back pain: an inception cohort in primary care physical therapy in The Netherlands. Spine. 2007 Mar 1; 32(5): 586-592.

[5] Steenstra IA, Verbeek JH, Heymans MW, Bongers PM. Prognostic factors for duration of sick leave in patients sick listed with acute low back pain: a systematic review of the literature. Occup Environ Med. 2005; 62(12): 851-860.

[6] Linton SJ. Occupational psychological factors increase the risk for back pain: a systematic review. J Occup Rehabil. 2001 Mar; 11(1): 53-66.

[7] Elfering A. Work-related outcome assessment instruments. Eur Spine J. 2006; 15(Suppl 1): S32-S43.

[8] Elfering A, Mannion AF. Epidemiology and risk factors of spinal disorders. In: Boos N, Aebi M, editors. Spinal disorders - Fundamentals of Diagnosis and Treatment. Berlin Heidelberg New York: Springer; 2008. p. 153-173.

[9] Hartvigsen J, Lings S, Leboeuf-Yde C, Bakketeig L. Psychosocial factors at work in relation to low back pain and consequences of low back pain; a systematic, critical review of prospective cohort studies. Occup Environ Med. 2004 Jan; 61(1): e2.

[10] Pincus T, Santos R, Breen A, Burton AK, Underwood M. A review and proposal for a core set of factors for prospective cohorts in low back pain: a consensus statement. Arthritis Rheum. 2008 Jan 15; 59(1): 14-24.

[11] Melloh M, Elfering A, Egli Presland C, Roeder C, Barz T, Rolli Salathe C, Tamcan O, Mueller U, Theis JC. Identification of prognostic factors for chronicity in patients with low back pain: a review of screening instruments. Int Orthop. 2009 Apr; 33(2): 301-313.

[12] Leeuw M, Houben RM, Severeijns R, Picavet HS, Schouten EG, Vlaeyen JW. Pain-related fear in low back pain: a prospective study in the general population. Eur J Pain. 2007; 11(3): 256-266.

[13] Leboeuf-Yde C. Body weight and low back pain. A systematic literature review of 56 journal articles reporting on 65 epidemiologic studies. Spine. 2000 Jan 15; 25(2): 226-237. 
[14] Linton SJ, Boersma K. Early identification of patients at risk of developing a persistent back problem: the predictive validity of the Orebro Musculoskeletal Pain Questionnaire. Clin J Pain. 2003 Mar-Apr; 19(2): 80-86.

[15] Waddell G, Burton AK. Occupational health guidelines for the management of low back pain at work: evidence review. Occup Med (Lond). 2001 Mar; 51(2): 124-135.

[16] Pincus T, Burton AK, Vogel S, Field AP. A systematic review of psychological factors as predictors of chronicity/disability in prospective cohorts of low back pain. Spine. 2002; 27(5): E109-E120.

[17] Leeuw M, Goossens ME, Linton SJ, Crombez G, Boersma K, Vlaeyen JW. The fear-avoidance model of musculoskeletal pain: current state of scientific evidence. J Behav Med. 2007; 30(1): 77-94

[18] Goldberg MS, Scott SC, Mayo NE. A review of the association between cigarette smoking and the development of nonspecific back pain and related outcomes. Spine. 2000; 25(8): 995-1014.

[19] Vuori IM. Dose-response of physical activity and low back pain, osteoarthritis, and osteoporosis. Med Sci Sports Exerc. 2001 Jun; 33(Suppl 6): S551-S586; discussion 609-610.

[20] Melloh M, Aebli N, Elfering A, Roder C, Zweig T, Barz T, Herbison P, Hendrick P, Bajracharya S, Stout K, Theis JC. Development of a screening tool predicting the transition from acute to chronic low back pain for patients in a GP setting: protocol of a multinational prospective cohort study. BMC Musculoskelet Disord. 2008 Dec 19; 9: 167.

[21] Airaksinen O, Brox JI, Cedraschi C, Hildebrandt J, KlaberMoffett J, Kovacs F, Mannion AF, Reis S, Staal JB, Ursin $\mathrm{H}$, Zanoli G. Chapter 4. European guidelines for the management of chronic nonspecific low back pain. Eur Spine J. 2006; 15(Suppl 2): S192-S300.

[22] Balague F, Mannion AF, Pellise F, Cedraschi C. Clinical update: low back pain. Lancet. 2007; 369(9563): 726-728.

[23] van Tulder M, Becker A, Bekkering T, Breen A, del Real MT, Hutchinson A, Koes B, Laerum E, Malmivaara A. Chapter 3. European guidelines for the management of acute nonspecific low back pain in primary care. Eur Spine J. 2006; 15(Suppl 2): S169-S191.

[24] Mielenz TJ, Garrett JM, Carey TS. Association of psychosocial work characteristics with low back pain outcomes. Spine. 2008 May 15; 33(11): 1270-1275.

[25] Woods V. Work-related musculoskeletal health and social support. Occup Med (Lond). 2005 May; 55(3): 177-189.

[26] Semmer NK, Udris I. Bedeutung und Wirkung von Arbeit. In: Schuler H, editor. Lehrbuch Organsationspsychologie. 4th ed. Bern: Huber; 2007. p. 157-195.

[27] Elfering A, Semmer NK, Schade V, Grund S, Boos N. Supportive colleague, unsupportive supervisor: the role of provider-specific constellations of social support at work in the development of low back pain. J Occup Health Psychol. 2002; 7(2): 130-140.

[28] Grotle M, Brox JI, Glomsrod B, Lonn JH, Vollestad NK. Prognostic factors in first-time care seekers due to acute low back pain. Eur J Pain. 2007 Apr; 11(3): 290-298.

[29] Bossuyt PM, Reitsma JB, Bruns DE, Gatsonis CA, Glasziou PP, Irwig LM, Lijmer JG, Moher D, Rennie D, de Vet HC. Towards complete and accurate reporting of studies of diagnostic accuracy: the STARD initiative. Fam Pract. 2004 Feb; 21(1): 4-10.

[30] Johanning E. Evaluation and management of occupational low back disorders. Am J Ind Med. 2000 Jan; 37(1): 94-111.

[31] Feuerstein M, Berkowitz SM, Haufler AJ, Lopez MS, Huang GD. Working with low back pain: workplace and individual psychosocial determinants of limited duty and lost time. Am J Ind Med. 2001 Dec; 40(6): 627-638. 